ture, solvent). All the information available on platinum metal catalysts modified by partial poisoning is assembled here.

The book is divided into six sections; on catalysis, equipment and conditions (54 pages); hydrogenation of unsaturated carbon-carbon bonds (57 pages); hydrogenation of nitrogen compounds (102 pages); carbonyl compounds (76 pages); aromatics (including nitrogen and oxygen heterocyclics) (83 pages), and a final section on hydrogenolysis (98 pages) including the Rosenmund reaction, dehalogenation and topics such as debenzylation. In the sections on the reactions of particular compounds, sufficient guidance is given to indicate the favoured methods of attacking new problems in the field. There are many empirical comparisons of catalysts in a given situation, but the book will not interest those who are concerned with the physical chemistry of catalysis, except as a source of fruitful systems for further work.

The catalytic reactions considered are all heterogeneous. The title is perhaps a little misleading, for the book deals not only with all the heterogeneous reactions, catalysed by platinum metals of hydrogen with substrates (mostly organic, though some inorganic molecules such as nitric oxide are described), but also with those reactions catalysed heterogeneously by solid derivatives of the platinum metals (for example, platinum oxide). There is no mention of the recent developments in homogeneous catalysis, which is perhaps a pity, for the relationship between the two areas of catalysis is likely to prove a very rich source of new organic reaction methods.

This book is beautifully printed, with very few errors indeed. It is copiously indexed. The price seems rather high. Dr Rylander, however, has produced a masterly account, obviously based on very deep experience, of the ways in which organic molecules may be caused to react with hydrogen, and this scholarly treatise should prove extremely useful to any chemist trying to solve a problem in hydrogenation.

R. D. GILLARD

\section{AMBER TO ELECTRICITY}

\section{Electricity and Matter}

By Norman Feather. Pp. viii +532. (Edinburgh: Edinburgh University Press, 1968.) 45s. net.

There is a lot written these days about the lack of students in the sciences, and almost as much speculation as to the causes. Bad teaching, the absolute difficulty of the subject, its inhumanity, all these have been and are being blamed. How nice then it is to find in one reasonably priced volume a positive inducement for the uncommitted to consider some of the beauty and fascination of a fundamental part of the physical sciences. Norman Feather, in his book on Electricity and Matter, takes a historical approach-but does not dodge the difficulties. He starts at the beginning: of course everybody knows about amber and how it may be induced to pick up bits of things, but here the telling of the tale seems to have a special authority. Classicists may like the link with amber's Greek name; nor will they-or the historiansbe disappointed if they read on-the mood so soon established is well maintained. Neither will those interested in human behaviour be unrewarded; the dilemmas, temptations, triumphs and false hopes all are there in the text, and in such a way as to interest without obscuring the essential story. A colleague who judges books on quantum mechanics by the ratio of words to formulae would find this book to his taste-there are formulae, but only those which are essential, and then thoy are used in such a way that they seem to sum up the explanatory writing in a succinct phrase. Indeed, someone innocent of mathematics reading the book could yet gain a great deal from it and might well be persuaded to learn more of such an obviously powerful technique.

The book is well produced and printed in clear easy-to. read type. The illustrations, of which there are perhaps less than one might have hoped for, are all line drawings; there are no photographs. 'The outer binding irritates in that it combines a "with-it" front and back cover (microphotographs? particle tracks? Rorschach test ?) with a traditional black and gold spine.

Although the book is probably intonded for under. graduates, it seems to me that it should also be in the sixth form library-and not necessarily only in the science section-true it becomes a bit advanced for most sixth formers, but at all times it encourages the student to read on and find out what happens next. On finishing it the main impulse is to rush out and buy, or at least borrow, the other two in the series. L. Molyneux

\section{SURVEY OF MATHEMATICS}

\section{Progress in Mathematics}

Vol. I: Mathematical Analysis. Edited by R. V. Gamkrelidze. Translated from the Russian. Pp. ix +246. (New York: Plenum Press, 1968.) \$15.

THIS is the first volume of a series which apparently is going to give survey papers of recent work. This volume contains three surveys, similar in general design, although varying somewhat in style and in the period covered. These surveys are written for mathematicians moderately familiar with the general field but not necessarily experts in the particular area surveyed. Concepts and notations are carefully defined and a detailed account of recent work is given. As far as possible, important results are given in full, in the form of theorems. Where this is not possible, because the results are too numerous or too involved, a brief indication of the result or the problem is given. Each survey article contains extensive references. Neither the text nor the bibliography claims encyclopaedic completeness but both are sufficiently detailed and systematic to serve as reliable guides. The thrce surveys are as follows.

"Operational Calculus" by Ditkin and Prudnikov (seventy-four pages, 486 references) deals with Miknsinski's operational calculus and related work, including operational calculus on a finite interval, semi-infinite and infinite interval, in several variables, and also of "Bessel opera. tors". Fantappié's analytic functionals, numerical methods, and some (but not many) applications are also mentioned.

"Spaces of Analytic Functions" by Khavin (ninetythree pages, 330 references) deals with applications of topological (in particular normed) vector space theory to problems concerning functions of one and several complex variables. The relation between generalized functions (distributions) and boundary values of analytic functions is included as are interpolation problems and "abstract" (= axiomatic) theories of functions, and vector-valued. analytic functions.

"Operational Differential Equations" by Nemytskii, Vainberg, and Gusarova (seventy-eight pages, 330 references) concerns differential equations in Banach (or sometimes locally convex vector) spaces. In the first part the operators involved are bounded, in which case the results are more complete, while in the second part the abstract Cauchy problem for linear equations involving an unbounded operator is considered.

The translation is moderately good, although the phrasing is sometimes awkward. Crude mistakes (such as "bounded" in place of "unbounded" on page 192) and misleading nomenclature ("group theoretic" on page 192 refers to the analytical theory of semi-groups, not to algebraic group theory) are rare. A. FRDÉLYI 